Research Paper

\title{
Age at First Birth and the Risk of Endometrial Cancer Incidence: A Pooled Analysis of Two Prospective Cohort Studies among Japanese Women
}

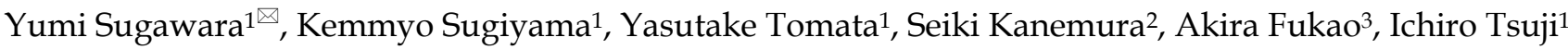 \\ 1. Division of Epidemiology, Department of Health Informatics and Public Health, Tohoku University School of Public Health, Graduate School of Medicine, \\ Sendai, Japan \\ 2. Division of Cancer Epidemiology and Prevention, Miyagi Cancer Center Research Institute, Natori, Japan \\ 3. Department of Public Health, Yamagata University Graduate School of Medicine, Yamagata, Japan \\ $\square$ Corresponding author: Yumi Sugawara, Ph.D. Division of Epidemiology, Department of Health Informatics and Public Health, Tohoku University School of \\ Public Health, Graduate School of Medicine, Sendai, Japan. 2-1, Seiryo-machi, Aoba-ku, Sendai, Miyagi, 980-8575, Japan. Phone: +81-22-717-8123; Fax: \\ +81-22-717-8125; E-mail: yumi1717@med.tohoku.ac.jp \\ () Ivyspring International Publisher. This is an open access article distributed under the terms of the Creative Commons Attribution (CC BY-NC) license \\ (https://creativecommons.org/licenses/by-nc/4.0/). See http://ivyspring.com/terms for full terms and conditions.
}

Received: 2018.03.23; Accepted: 2018.08.26; Published: 2018.10.27

\begin{abstract}
Background: Age at first birth has been increasing among women in developed countries. Meanwhile, endometrial cancer has also been increasing worldwide, being one of the most common female hormone-related cancers. The purpose of this study was to examine the association between age at first birth and the risk of endometrial cancer among Japanese women, and to examine the hypothesis that the recent increase in endometrial cancer incidence can be partly explained by the trend for increasing age at first birth.

Methods: We conducted a pooled analysis of two prospective studies among residents in Miyagi Prefecture in rural northern in Japan. The Miyagi Cohort Study started in 1990 and included 21,455 parous women. The Ohsaki Cohort Study started in 1994 and included 17,287 parous women. The subjects responded to a self-administrated questionnaire including reproductive factors such as age at first birth. Incident cases of cancer were identified through linkage to the Miyagi Prefectural Cancer Registry, which covers the study area.

Results: In a consortium of two prospective studies with 598,933 person-years, we identified 105 incident case of endometrial cancer. Compared with women aged 22 years or less at first birth, multivariate hazard ratios (HRs) and $95 \%$ confidence intervals $(\mathrm{Cls})$ for the risk of endometrial cancer were $0.79(0.49-1.26)$ for women aged 23 to 25 years at first birth, and $0.53(0.28-1.00)$ for those aged 26 years and older ( $p$-trend $<0.05$ ).

Conclusion: This pooled analysis of two prospective studies does not support the hypothesis that the recent increase in the incidence of endometrial cancer can be partly explained by the increase in the age at first birth.
\end{abstract}

Key words: age at first birth; endometrial cancer; incidence; Japanese women; prospective cohort study

\section{Introduction}

The age of women at first birth has been increasing in developed countries [1]. For example, in Japanese women the average age at first birth increased by 5.1 -years, from 25.6 to 30.7 years, in the period between 1970 and 2015 [2]. Epidemiologic studies have examined the association between age at first birth and health-related factors such as obesity, hypertension, diabetes mellitus and cancer in parous women [3-5]. Previous studies have agreed that breast cancer risk increases with age at first birth [6-8], and the mechanism responsible is considered to be exposure to excess endogenous estrogen $[9,10]$. 
By contrast, the relationship between age at first birth and the risk of endometrial cancer, one of the most common female hormone-related cancers, remains unclear. As the incidence of endometrial cancer is currently increasing worldwide $[8,11]$, it is reasonable to hypothesize that this can be partly explained by the recent increase in age at first birth.

The results of eight prospective cohort studies examining the association between age at first birth and the risk of endometrial cancer have not been consistent. One study from Sweden found a positive association between age at first birth and the risk of endometrial cancer incidence [12], whereas three studies from China, Sweden, and the USA have reported an inverse association [13-15]. The remaining four studies from Norway, the USA, Sweden, and Germany have reported no association [16-19]. Seven of these eight studies examined American or European women. To date, the Chinese study is the only one to have examined population from Asia, where life-style, dietary habits, and hormonal factors differ from those in Western countries. In addition, some of the previous studies were limited because of insufficient adjustment for potential confounding factors. Accordingly, it is still premature to draw any conclusion about the relationship between age at first birth and the risk of endometrial cancer.

The purpose of the present study was to examine the association between age at first birth and the risk of endometrial cancer among Japanese women, and to examine the hypothesis that the recent increase in the incidence of endometrial cancer can be partly explained by the trend for increasing age at first birth.

\section{Materials and Methods}

\section{Study population}

For this analysis, we conducted a pooled analysis of two population-based prospective cohort studies, the Miyagi Cohort Study and the Ohsaki Cohort Study in rural northern Japan. The Study design for the two cohort studies have been described in detail elsewhere [20-22]. Briefly, for the Miyagi Cohort Study, we delivered a self-administered questionnaire from June to August 1990 to 26,642 women aged 40-64 years who were living in 14 municipalities, which were randomly selected from 62 municipalities in Miyagi Prefecture, northeastern Japan. Usable questionnaires were returned from 24,769 subjects (92.9\%). For the Ohsaki Cohort Study, between October and December 1994, we delivered a self-administered questionnaire to 28,515 women who were 40-79 years old and living in the catchment area of Ohsaki Public Health Center, a local government agency that provides preventive health services for the residents of 14 municipalities in Miyagi Prefecture. Usable questionnaires were returned from 27,133 subjects $(95.2 \%)$. The questionnaire included items on demographic and lifestyle factors, and reproductive factors such as age at first birth.

The study protocols for the two cohorts were approved by the Institutional Review Board of Tohoku University Graduate School of Medicine. We considered the return of a signed self-administered questionnaire to imply each subject's consent to participate in the study.

We excluded from our analysis women who had moved from the study area before the start of follow-up $(\mathrm{N}=454)$, and one woman whose date of cancer diagnosis was missing in Ohsaki. Also, during the process of data pooling of the two cohorts, we identified 2,869 individuals who participated in both cohorts. Thus, we eliminated the data of these individuals only from the Ohsaki Cohort Study, whose baseline was after that of the Miyagi Cohort Study. In addition, for the present analysis, we excluded women who had a history of cancer at the baseline $(\mathrm{N}=2,174)$, those who were nulliparous $(\mathrm{N}=1,196)$, those for whom questionnaire data on pregnancy were missing $(\mathrm{N}=5,697)$, and those who had failed to enter questionnaire data for age at first birth $(\mathrm{N}=769)$. Thus, 38,742 women were included in the study cohort.

\section{Exposure data}

The self-administrated questionnaire items inquiring about medical history, family history, job status, marital status, education, and health-related lifestyle factors such as smoking status, alcohol consumption, and time spent walking per day. It also included reproductive factors (menopausal status, age at menarche, age at first birth, number of births, history of oral contraceptive use, history of hormone replacement therapy, and lactation). We divided the participants into tertiles based on the age at first birth (years): $\leq 22$ years, $23-25$ years, $\geq 26$ years.

\section{Follow-up}

Follow-up was conducted from June1990 to 31 December 2010, for the Miyagi Cohort Study, and from 1 June 1995 to 31 March 2008, for the Ohsaki Cohort Study. The end point of our analysis was the date of diagnosis of endometrial cancer, death, emigration, or end of the follow-up period, whichever occurred first. In order to follow -up the subjects for migration and mortality, we collected data from the Residential Records to identify subjects who had either died or emigrated during the observation period. We discontinued the follow-up of participants 
who had moved from the study municipalities, because we were unable to review the Residential Records from outside the study area. The portions of subjects who were lost to follow-up were $12.3 \%$ for the two prospective studies. We also ascertained incident cases of cancer via computer link with the Miyagi Prefectural Cancer Registry, which covers both the areas of both the Miyagi cohort and the Ohsaki cohort. The Miyagi Prefectural Cancer Registry is one of oldest and most accurate population-based cancer registries in Japan. Cancer cases are registered from clinics and hospitals (inpatients and outpatients), radiology and pathology departments, autopsy records, mass screening records and death certificates. In this cancer registry for the period 2003-2007, 7.4\% death certificate only (DCO) registered deaths among women were due to endometrial cancer [11]. Cancer incidence data were coded according to the International Classification of Diseases for Oncology, 3nd version (ICD-O-3) as endometrial cancer (C54.0-C54.9).

\section{Statistical analysis}

We prospectively counted person-years of follow-up for each subject from the beginning until the end of follow-up. Next, we used Cox proportional hazards regression analysis to estimate the hazard ratios (HRs) and 95\% confidence intervals (CIs) for the incidence of endometrial cancer according to the lowest category of age at first birth as the reference group, after adjustment for potential confounders. Further, we divided women who were the highest category of age at first birth into two categories, and examined the association between age at first birth and endometrial cancer incidence. Also, to consider the effect of reverse causality, we carried out sensitivity analysis excluding cases of endometrial cancer that occurred in the first 3 years. We considered for the following variables as potential confounders: age at baseline (continuous variable), body mass index (BMI) in $\mathrm{kg} / \mathrm{m}^{2}(\leq 18.5,18.5-24.9$, $\geq 25.0$ ), family history of cancer (yes, no), education (high school or less, college/university or higher), smoking status (never smoked, ever smoked), alcohol consumption (never drank alcohol, ever drank alcohol), time spent walking ( $<1.0$ hour/day, $\geq 1$ hour/day), age at menarche (continuous variable), number of births (continuous variable), history of oral contraceptive use (yes, no). These items were chosen as covariates because previous studies had shown that they are factors related to the incidence of endometrial cancer [7, 8]. Education level, marital status and health-related factors (BMI, smoking status, and alcohol consumption) might be associated with both age at first birth and the risk of endometrial cancer, because they would affect the timing of births. There is some evidence suggesting that physical activity reduces the risk of endometrial cancer [8]. As is the case for other hormone-related cancers, the risk of endometrial is influenced by lifetime exposure to plasma estrogen. Thus, reproductive factors such as age at menarche, number of births, and history of oral contraceptive use were also strongly associated with both age at first birth and the risk of endometrial cancer.

The $P$ values for the test of linear trend ( $P$-trend) were calculated using median value of each category of age at first birth. The significance of multiplicative interactions between age at first birth and all confounders was examined using cross-product terms in the regression model.

According to the World Cancer Research Fund and American Institute for Cancer Research (WCRF/AICR), being overweight or obese is a convincing risk factor for endometrial cancer [8]. We also conducted stratified analysis by age and BMI at the time of the baseline survey, because we considered that postpartum weight change would be a factor related to parous women being overweight or obese.

Furthermore, we conducted analysis stratified by age at menarche ( $<16$ years or $\geq 16$ years), number of births (1-2 births, $\geq 3$ births), and age at menopause ( $<50$ years or $\geq 50$ years), because these factors may affect cancer risk by influencing the levels of estrogens and other sex hormones [8-10].

All $P$ values were two-sided, and differences at $P<0.05$ were considered statistically significant. All statistical analyses were performed using the SAS statistical software package, version 9.4 (SAS Institute Inc, Cary, NC, USA).

\section{Results}

Table 1 compares the baseline characteristics of the study participants according to the categories age at first birth. Women who were older at first birth in the Miyagi study tended to be better educated, younger at menarche, and have a lower number of births, while such women in the Ohsaki Cohort tended to be older, postmenopausal and more likely to be employed. Furthermore, women with a higher age at first birth in both cohorts were also less likely to have used oral contraceptives. There were no inter-group differences in mean body mass index, family history of cancer, or history of hormone replacement therapy.

During follow-up, 105 incident cases of endometrial cancer were observed. Table 2 shows the HRs and 95\% CIs for the risks of endometrial cancer incidence according to age at first birth. Multivariate 
analysis showed that higher age at first birth was significantly associated with a decreased risk of endometrial cancer incidence $(P$-trend $<0.05)$. Compared with participants who had first given birth before the age of 23 years, the multivariate HR
$(95 \% \mathrm{CI})$ for endometrial cancer incidence was 0.79 (0.49-1.26) for participants who were 23 to 25 years old at the time of first birth, and $0.53(0.28-1.00)$ for those who were 26 years old or more.

Table 1. Characteristics of participants according to two prospective studies $(n=38,742)$

\begin{tabular}{|c|c|c|c|c|c|c|}
\hline & \multicolumn{6}{|c|}{ Age at first delivery } \\
\hline & \multicolumn{3}{|c|}{ Miyagi $(n=21,455)$} & \multicolumn{3}{|c|}{ Ohsaki $(n=17,287)$} \\
\hline & $\leq 22$ & $23-25$ & $26 \leq$ & $\leq 22$ & $23-25$ & $26 \leq$ \\
\hline Number of subjects & 6,084 & 9,855 & 5,516 & 6,058 & 7,604 & 3,625 \\
\hline Age, years (SD) & $52.3(7.6)$ & $52.0(7.4)$ & $52.1(7.3)$ & $60.6(10.9)$ & $60.0(9.9)$ & $61.0(10.2)$ \\
\hline Body mass index, $\mathrm{kg} / \mathrm{m}^{2}(\mathrm{SD})$ & $24.0(3.1)$ & $23.8(3.1)$ & $23.5(3.2)$ & $24.0(3.5)$ & $23.8(3.3)$ & $23.5(3.4)$ \\
\hline Family history of cancer (\%) & 27.9 & 27.9 & 28.2 & 32.2 & 33.4 & 33.5 \\
\hline \multicolumn{7}{|l|}{ Education (\%) } \\
\hline High school or less & 85.1 & 82.1 & 77.9 & 89.8 & 88.3 & 83.5 \\
\hline College / university or higher & 8.1 & 11.8 & 16.2 & 5.0 & 7.5 & 12.5 \\
\hline Employed (\%) & 52.8 & 51.1 & 45.2 & 37.0 & 38.1 & 30 \\
\hline Ever smoked (\%) & 9.7 & 6.7 & 9.0 & 9.0 & 6.7 & 10.1 \\
\hline Ever drank alcohol (\%) & 25.9 & 22.7 & 25.1 & 23.4 & 21.9 & 24.7 \\
\hline \multicolumn{7}{|l|}{ Time spent walking (\%) } \\
\hline$<1$ hour/day & 45.0 & 48.8 & 54.4 & 49.0 & 50.6 & 58.9 \\
\hline$\geq 1$ hour/day & 45.0 & 42.3 & 37.2 & 41.8 & 40.9 & 33.9 \\
\hline \multicolumn{7}{|l|}{ Menopausal status (\%) } \\
\hline premenopausal & 39.2 & 40.6 & 40.3 & 20.3 & 18.9 & 18.5 \\
\hline postmenopausal & 52.7 & 51.5 & 52.9 & 68.6 & 70.6 & 73 \\
\hline \multicolumn{7}{|l|}{ Age at menarche $(\%)$} \\
\hline$\leq 12$ years & 6.8 & 7.7 & 8.8 & 1.7 & 2.3 & 3.2 \\
\hline $13-15$ years & 57.7 & 57.2 & 56.9 & 28.7 & 29.0 & 30.2 \\
\hline$\geq 16$ years & 25.4 & 25.1 & 24.3 & 20.9 & 23.3 & 24.5 \\
\hline \multicolumn{7}{|l|}{ Number of deliveries (\%) } \\
\hline 1 & 3.0 & 4.9 & 16.7 & 2.7 & 4.2 & 15.0 \\
\hline 2 & 32.5 & 43.9 & 50.0 & 26.9 & 37.1 & 44.0 \\
\hline$\geq 3$ & 64.5 & 51.2 & 33.3 & 70.4 & 58.7 & 41.1 \\
\hline \multicolumn{7}{|c|}{ History of oral contraceptive drug use (\%) } \\
\hline yes & 7.3 & 5.2 & 4.0 & 5.0 & 4.2 & 3.2 \\
\hline \multicolumn{7}{|c|}{ History of hormone replacement therapy (\%) } \\
\hline yes & 6.0 & 6.1 & 6.4 & 6.7 & 7.2 & 8.1 \\
\hline
\end{tabular}

SD; standard deviation.

Table 2. Cox Proportional Hazard Ratios (HRs) of endometrial cancer incidence according to age at first birth; a pooled analysis of two prospective studies

\begin{tabular}{|c|c|c|c|c|}
\hline & \multicolumn{4}{|c|}{ Age at first birth } \\
\hline & $\leq 22$ & $23-25$ & $\geq 26$ & $p$-trenda \\
\hline Number of participants & 12,142 & 17,459 & 9,141 & \\
\hline Person-years & 182,309 & 273,060 & 143,564 & \\
\hline Number of cases & 38 & 48 & 19 & \\
\hline Incidence rate $b$ & 20.84 & 17.58 & 13.23 & \\
\hline Crude HR (95\% CI) & 1.00 (ref) & $0.84(0.55-1.28)$ & $0.63(0.36-1.09)$ & 0.09 \\
\hline Age adjusted HR (95\% CI) & 1.00 (ref) & $0.84(0.55-1.29)$ & $0.63(0.36-1.09)$ & 0.10 \\
\hline Multivariate HRc $(95 \%$ CI) & 1.00 (ref) & $0.79(0.49-1.26)$ & $0.53(0.28-1.00)$ & $<0.05$ \\
\hline Multivariate HRd(95\% CI) & 1.00 (ref) & $0.73(0.44-1.19)$ & $0.47(0.23-0.93)$ & $<0.05$ \\
\hline
\end{tabular}

P-trenda were calculated using median value of each category of age at first birth

Incidence rate ${ }^{b}$ were expressed as number per 100,000 person-years.

The multivariate $\mathrm{HR}^{\mathrm{c}}$ has been adjusted for age at baseline(continuous variable), body mass index in $\mathrm{kg} / \mathrm{m}^{2}(<18.5,18.5-24.9$, or $\geq 25.0)$, family history of cancer (yes or no) smoking status (never smoked, or ever smoked), alcohol drinking (never drank alcohol, or ever drank alcohol), education (high school or less, or college/university or higher), time spent walking ( $<1$ hour/day, or $\geq 1$ hour/day), age at menarche (continuous variable), number of births (continuous variable), history of oral contraceptive use (yes or no). The multivariate $\mathrm{HR}^{\mathrm{d}}$ were calculated excluding case of endometrial cancer during first 3 years from Multivariate-adjusted HRc.

We also analyzed the association between age at first birth and the incidence of endometrial cancer when divided women who were 26 years old or more at the time of first birth into two categories (26-29 years, or $\geq 30$ years). Similarly, we found that women who were old at first birth had a significantly decreased risk of endometrial incidence ( $P$-trend $<0.05)$. The multivariate HR $(95 \% \mathrm{CI})$ for endometrial 
cancer incidence was 0.44 (0.20-0.94) for participants who were 26 to 29 years old at the time of first birth, and $0.59(0.20-2.00)$ for those who were 30 years old or more.

Also, after exclusion of incident cases that occurred within the first 3 years of follow-up, but the results remained unchanged.

We observed similar results between the Miyagi Cohort Study and the Ohsaki Cohort Study. The result of multivariate analysis also was significantly associated with a decreased risk of endometrial cancer incidence.

Table 3 shows the resulting HRs and 95\% CIs for endometrial cancer incidence according to age at first birth, stratified by age at baseline, BMI and reproductive factors (i.e., age at menarche, number of births, and age at menopause) at the baseline. We consistently observed an inverse association between an older age at birth and the risk of endometrial cancer. However, we did not find any statistically significant effect modifications between age at first birth and any of the covariates.

For women who were 26 years old or more at the time of first birth, the multivariate HRs (95\% CIs) for endometrial cancer incidence were 0.67 (0.29-1.53) for BMI $<25$ and $0.38 \quad(0.13-1.05)$ for BMI $\geq 25$. Additionally, we obtained similar results for age at menarche (HR 0.71, 95\% CI: 0.38-1.35 for women under 16 years old, and HR 0.29, 95\% CI: 0.08-1.12 for those over 16 years old), number of births (HR 0.51, 95\% CI: $0.22-1.20$ for 1-2 births, and HR 0.62, 95\% CI: 0.23-1.65 for 3 births), and age at menopause (HR 0.32, 95\% CI: 0.04-2.84 for under 50 years, and HR 0.61, 95\% CI: $0.23-1.63$ for over 50 years). However, this may have been a chance finding, because the number of endometrial cancers was very small.

Table 3. Hazard ratio (HR) for endometrial cancer incidence according to age at first birth stratified by age, BMI, and reproductive factors

\begin{tabular}{|c|c|c|c|c|c|}
\hline & \multicolumn{5}{|c|}{ Age at first birth } \\
\hline & $\leq 22$ & $23-25$ & $\geq 26$ & $p$-trenda & $p$-interaction \\
\hline \multicolumn{6}{|l|}{ Age at baseline survey } \\
\hline$<50$ years & & & & & 0.420 \\
\hline Person-years & 59,134 & 90,131 & 46,577 & & \\
\hline Number of cases & 13 & 12 & 5 & & \\
\hline Incidence rate ${ }^{b}$ & 21.98 & 13.31 & 10.74 & & \\
\hline Multivariate HRc $(95 \%$ CI) & 1.00 (ref) & $0.67(0.28-1.59)$ & $0.59(0.19-1.81)$ & 0.34 & \\
\hline Multivariate $\mathrm{HR}^{\mathrm{d}}(95 \% \mathrm{CI})$ & 1.00 (ref) & $0.61(0.25-1.49)$ & $0.59(0.19-1.84)$ & 0.34 & \\
\hline$\geq 50$ years & 8563 & & & & \\
\hline Person-years & 123,174 & 182,929 & 96,987 & & \\
\hline Number of cases & 25 & 36 & 14 & & \\
\hline Incidence rateb & 20.30 & 19.68 & 14.43 & & \\
\hline Multivariate HRc $(95 \%$ CI) & 1.00 (ref) & $0.85(0.49-1.48)$ & $0.51(0.24-1.12)$ & 0.09 & \\
\hline Multivariate $\mathrm{HRd}^{\mathrm{d}}(95 \% \mathrm{CI})$ & 1.00 (ref) & $0.80(0.44-1.45)$ & $0.42(0.17-1.02)$ & 0.05 & \\
\hline \multicolumn{6}{|l|}{ BMI at baseline survey } \\
\hline$<25$ & & & & & 0.93 \\
\hline Person-years & 122,183 & 190,156 & 103,538 & & \\
\hline Number of cases & 18 & 26 & 10 & & \\
\hline Incidence rateb & 14.73 & 13.67 & 9.66 & & \\
\hline Multivariate HRc $(95 \%$ CI) & 1(ref) & $0.81(0.41-1.58)$ & $0.67(0.29-1.53)$ & 0.34 & \\
\hline Multivariate $\mathrm{HR}^{\mathrm{d}}(95 \% \mathrm{CI})$ & 1.00 (ref) & $0.70(0.35-1.39)$ & $0.55(0.22-1.33)$ & 0.18 & \\
\hline \multicolumn{6}{|l|}{$\geq 25$} \\
\hline Person-years & 60,126 & 82,904 & 40,026 & & \\
\hline Number of cases & 20 & 22 & 9 & & \\
\hline Incidence rate ${ }^{b}$ & 33.26 & 26.54 & 22.49 & & \\
\hline Multivariate HRc $(95 \%$ CI $)$ & 1.00 (ref) & $0.78(0.40-1.49)$ & $0.38(0.13-1.05)$ & 0.06 & \\
\hline Multivariate $\mathrm{HR}^{\mathrm{d}}(95 \% \mathrm{CI})$ & 1.00 (ref) & $0.76(0.38-1.54)$ & $0.36(0.11-1.12)$ & 0.07 & \\
\hline \multicolumn{6}{|l|}{ Age at menarche } \\
\hline$<16$ years & & & & & 0.44 \\
\hline Person-years & 139,358 & 206,701 & 109,046 & & \\
\hline Number of cases & 28 & 36 & 16 & & \\
\hline Incidence rate ${ }^{b}$ & 20.09 & 17.42 & 14.67 & & \\
\hline Multivariate HRc $(95 \%$ CI) & 1.00 (ref) & $0.84(0.51-1.39)$ & $0.71(0.38-1.35)$ & 0.30 & \\
\hline Multivariate $\mathrm{HR}^{\mathrm{d}}(95 \% \mathrm{CI})$ & 1.00 (ref) & $0.79(0.46-1.34)$ & $0.61(0.30-1.24)$ & 0.16 & \\
\hline \multicolumn{6}{|l|}{$\geq 16$ years } \\
\hline Person-years & 42,950 & 66,359 & 34,518 & & \\
\hline Number of cases & 10 & 12 & 3 & & \\
\hline Incidence rate $e^{b}$ & 23.28 & 18.08 & 8.69 & & \\
\hline Multivariate HRc $(95 \%$ CI $)$ & 1.00 (ref) & $0.72(0.31-1.67)$ & $0.29(0.08-1.12)$ & 0.07 & \\
\hline Multivariate $\mathrm{HR}^{\mathrm{d}}(95 \% \mathrm{CI})$ & 1.00 (ref) & $0.76(0.31-1.84)$ & $0.23(0.05-1.13)$ & 0.06 & \\
\hline Number of births & & & & & \\
\hline
\end{tabular}




\begin{tabular}{|c|c|c|c|c|c|}
\hline & \multicolumn{5}{|c|}{ Age at first birth } \\
\hline & $\leq 22$ & $23-25$ & $\geq 26$ & $p$-trenda & $p$-interaction \\
\hline 1-2 births & & & & & 0.83 \\
\hline Number of participants & 61,233 & 127,094 & 92,556 & & \\
\hline No. of cases & 15 & 19 & 14 & & \\
\hline Incidence rate ${ }^{b}$ & 24.50 & 14.95 & 15.13 & & \\
\hline Multivariate HRc $(95 \%$ CI) & 1.00 (ref) & $0.69(0.34-1.43)$ & $0.51(0.22-1.20)$ & 0.13 & \\
\hline Multivariate $\mathrm{HR}^{\mathrm{d}}(95 \% \mathrm{CI})$ & 1.00 (ref) & $0.64(0.30-1.38)$ & $0.41(0.16-1.06)$ & 0.07 & \\
\hline \multicolumn{6}{|l|}{$\geq 3$ births } \\
\hline Person-years & 121,076 & 145,966 & 51,008 & & \\
\hline Number of cases & 23 & 29 & 5 & & \\
\hline Incidence rate ${ }^{b}$ & 19.00 & 19.87 & 9.80 & & \\
\hline Multivariate HRc $(95 \% \mathrm{CI})$ & 1.00 (ref) & $0.91(0.50-1.67)$ & $0.62(0.23-1.65)$ & 0.36 & \\
\hline Multivariate $\mathrm{HR}^{\mathrm{d}}(95 \% \mathrm{CI})$ & 1.00 (ref) & $0.82(0.43-1.55)$ & $0.64(0.24-1.74)$ & 0.35 & \\
\hline \multicolumn{6}{|l|}{ Age at menopause } \\
\hline$<50$ years & & & & & 0.73 \\
\hline Person-years & 47,880 & 69,449 & 34,548 & & \\
\hline Number of cases & 5 & 8 & 1 & & \\
\hline Incidence rate ${ }^{b}$ & 10.44 & 11.52 & 2.89 & & \\
\hline Multivariate HRc $(95 \%$ CI) & 1.00 (ref) & $1.17(0.38-3.62)$ & $0.32(0.04-2.84)$ & 0.31 & \\
\hline Multivariate $\mathrm{HR}^{\mathrm{d}}(95 \% \mathrm{CI})$ & 1.00 (ref) & $1.01(0.32-3.24)$ & $0.31(0.04-2.83)$ & 0.34 & \\
\hline \multicolumn{6}{|l|}{$\geq 50$ years } \\
\hline Person-years & 46,464 & 70,277 & 40,247 & & \\
\hline Number of cases & 12 & 19 & 7 & & \\
\hline Incidence rate ${ }^{b}$ & 25.83 & 27.04 & 17.39 & & \\
\hline Multivariate HRc $(95 \% \mathrm{CI})$ & 1.00 (ref) & $1.03(0.48-2.18)$ & $0.61(0.23-1.63)$ & 0.12 & \\
\hline Multivariate HRd $(95 \%$ CI) & 1.00 (ref) & $0.95(0.42-2.15)$ & $0.43(0.13-1.42)$ & 0.17 & \\
\hline
\end{tabular}

P-trenda were calculated using median value of each category of age at first birth. Incidence rate ${ }^{b}$ were expressed as number per 100,000 person-years.

The multivariate HRc has been adjusted for age ( $<50$ years or $\geq 50$ years), body mass index in $\mathrm{kg} / \mathrm{m}^{2}(<25.0$ or $\geq 25.0$ ), family history of cancer (yes or no), smoking status (never smoked, or ever smoked), alcohol drinking (never drank alcohol, or ever drank alcohol), education (high school or less, or college/university or higher), time spent walking ( $<1$ hour/day, or $\geq 1$ hour/day), age at menarche ( $<16$ years or $\geq 16$ years), number of births ( $<3$ births or $\geq 3$ births), history of oral contraceptive use (yes or no).

\section{Discussion}

The age of women at the time of first birth has been increasing in the developed countries, and its health impact upon parous women has been examined worldwide. The present study demonstrated an inverse association between age at first birth and the risk of endometrial cancer incidence. Stratified analysis indicated that this inverse association was valid, irrespective of whether the subjects were younger or older, whether or not they were overweight, whether they were younger or older age at menarche, whether they had 1-2 births or more, and whether they were younger or older at menopause. In addition, we also found that women who were old at first birth had a significantly decreased risk of endometrial incidence, even after exclusion of incident cases that occurred within the first 3 years of follow-up.

Furthermore, we thought that incomplete pregnancies may have influenced the association between age at first birth and endometrial cancer incidence. To assess whether the findings are consistent when women with no live births are included as the referent group, we have recalculated the association between age at first birth and the risk of endometrial cancer including the 44 women who had no live births. As a result, our findings were the same even when we included or excluded women with no live birth in the multivariate analysis (data not shown).

Therefore, our findings do not support the hypothesis that the recent increase in endometrial cancer incidence can be partly explained by the increasing in the age at first birth.

Eight prospective cohort studies have already examined the association between age at first birth and the risk of endometrial cancer [12-19]. One study concluding that a higher age at first birth was associated with an increased the risk of endometrial cancer adjusted for only parity in the analysis [12]. Although three prospective studies have reported an inverse association between age at first birth and the risk of endometrial cancer, adjustment for potential confounding factors was insufficient [13-15]. For example, in the Shanghai Textile Industry Bureau (STIB) examination, women with a higher age at first birth had a decreased risk of endometrial cancer incidence ( $p$-trend=0.03) [13]. The remaining four studies found no association between age at first birth and the risk of endometrial cancer, but all were conducted in USA and Europe, where oral contraceptives and hormone replacement therapy are used more frequently than in Japan [16-19]. In these areas, not only endogenous but also exogenous hormonal factors would affect the risk of endometrial cancer, 
thus leading to underestimation of the effect of endogenous hormonal factors. In order to examine the effect of endogenous hormonal factors accurately, it would be appropriate to study a population with low use of oral contraceptives or hormone replacement therapy, as is the case in Japan. Here, we found that an older age at the time of first birth was inversely associated with the risk of endometrial cancer incidence, the association being independent of the number of births and age at menopause. Our result is consistent with three epidemiologic studies that found an inverse association between age at first birth and the risk of endometrial cancer [13-15].

The risk of endometrial cancer is related to stimulation to the endometrium by estrogen, as explained by the "unopposed estrogen hypothesis" [8-10]. Thus, endometrial cancer risk is increased in women who have high plasma estrogen that is unopposed by progesterone [8-10]. During pregnancy when progesterone production is high, it reduces the length of time that endometrium is exposed estrogen. Additionally, women who were older at first birth might be have good hormonal cycle and they were less likely to be infertility and polycystic ovary syndrome that have been reported to be risk factors for endometrial cancer.

One study from Sweden has reported that women who were older at first birth had an increased risk of endometrial cancer [12]. By contrast, three studies from China, Sweden, and the USA have reported that women who were old at first birth had a significantly decreased risk of endometrial incidence [13-15]. Our result is consistent with the latter three studies.

The reasons for the discrepancy between our finding and the Sweden cohort study might have been an "immortal time bias". In the Sweden cohort study, the follow-up period was from the date of first birth to the end of follow-up, whereas the follow-up period in the present study was from the age at inclusion in the cohort to the end of follow-up. The subjects were healthy women who had no events, and there would have been a possible "immortal time bias" for older women who had been included in the cohort at the baseline. However, when we stratified our analyses according to age at the baseline $(<50$ years or $\geq 50$ years), the result did not change (Table 3$)$. This suggests that any "immortal time bias" would have been less likely to affect the association between age at first birth and the risk of endometrial cancer.

Additionally, the result of our study raises the possibility that women who are older at the time of first birth would likely to be older at the time of their final birth, thus possibly decreasing the risk of endometrial cancer. In the Nurses' health study, inverse association was found, and this was diminished after adjustment for the age at the time of final birth [14]. Similarly, a study from Sweden found that women in whom more than 10 years had elapsed between their first and last births had a decreased risk of endometrial cancer [15]. In the present study, however, we did not clarify whether age at first birth or at the last birth had a stronger impact on the incidence of endometrial cancer, because we did not ask women to state their age at the time of their last birth.

The major strengths of our study included its prospective design, and adjustment for many potential confounding factors. Second, the response rate was high $(94.1 \%)$ and the selection bias was small. Third, the internal validity of this study was extremely high, because the age-standardized endometrial incidence rate in the study area was almost the same as that for the Japanese population as a whole [11]. Furthermore, our results would not have been significantly influenced by exogenous hormone use and smoking.

This study also had some limitations. First, the statistical power was low, because the number of cause of endometrial cancer was low. However, the association between age at first birth and the risk of endometrial cancer remained essentially unchanged when we conducted separate analyses for obesity and reproductive factors. Second, we collected information on age at first birth was ascertained using a self-reported questionnaire at baseline. Therefore, some misclassification would have been inevitable, and the reported hazard ratios would have been closer to a null value than a true relationship. Third, we had no data on the age at last birth, and thus could not examine this factor in relation to the risk of endometrial cancer [7, 8-10, 23-25]. Moreover, we did not collect information about the menstrual cycle. However, this would have had little influence on our results, because our findings were the same when we conducted separate analyses for reproductive factors such as age at menarche, the number of births, and age at menopause. Forth, several risk factors that affect the risk of endometrial cancer may have been modified during follow-up. However, we thought that there were no differences among the groups.

In conclusion, this pooled analysis of two prospective studies conducted among Japanese women does not support the hypothesis that the recent increase in the incidence of endometrial cancer can be partly explained by the increase in the age at first birth. Although age at first birth is undoubtedly increasing among women who live in developed countries, we suggest that this is un-likely to contribute to the increase in endometrial cancer risk. 


\section{Supplementary Material}

Appendix 1. Cox Proportional Hazard Ratios (HRs) of endometrial cancer incidence according to age at first birth; a pooled analysis of two prospective studies. http://www.jcancer.org/v09p4422s1.pdf

\section{Acknowledgements}

The authors gratefully acknowledgements the assistance of Yoshiko Nakata and Mami Takahashi, Tohoku University Graduate School of Medicine.

\section{Funding}

This study was supported by the National Cancer Center Research and Development Fund (27A-4, 30-A-15).

\section{Authorship Contribution}

YS participated in the design of the study, analysis and interpretation of data, and wrote the manuscript. KS participated in its design, conducted research, and helped the statistical analysis. YT, SK, and AF participated in its design, conducted research, and helped to draft the manuscript. IT participated in its design, and provided commentaries to the manuscript text. All authors read and approved the final manuscript.

\section{Competing Interests}

The authors have declared that no competing interest exists.

\section{References}

[1] Mathews TJ, Hamilton BE. Delayed Childbearing: More Women Are Having Their First Child Later in Life. NCHS Data Brief. 2009;21: 1-8.

[2] Ministry of Health, Labor and Welfare, Japan. Vital Statistics of Japan. 2015. "4-19: Trends in mean age of mother by live birth order: Japan". (http://www.e-stat.go.jp). (Accessed Mar 14, 2018)

[3] Ziadeh S, Yahaya A. Pregnancy outcome at maternal age 40 and older. Arch Gynecol Obstet. 2001;265: 30-33.

[4] Joseph KS, Allen AC, Dodds L, Turner LA, Scott H, Liston R. The perinatal effects of delayed childbearing. Obstet Gynecol. 2005;105: 1410-1418.

[5] Merrill RM, Fugal S, Novilla LB, Raphael MC. Cancer risk associated with early and late maternal age at first birth. Gynecol Oncol. 2005;96: 583-593.

[6] Sweeney C, Blair CK, Anderson KE, Lazovich D, Folsom AR. Risk factors for breast cancer in elderly women. Am J Epidemiol. 2004;160: 868-875.

[7] Li CI, Littman AJ, White E. Relationship between age maximum height is attained, age at menarche, and age at first full-term birth and breast cancer risk. Cancer Epidemiol Biomarkers Prev. 2007;16: 2144-2149.

[8] World Cancer Research Fund / American Institute for Cancer Research. Endometrial Cancer 2013 Report. Food, Nutrition, Physical Activity, and the Prevention of Endometrial Cancer: American Institute for Cancer Research. 2013.

[9] Henderson BE, Ross RK, Pike MC, Casagrande JT. Endogenous hormones as a major factor in human cancer. Cancer Res.1982;42: 3232-3239.

[10] Key TJ, Verkasalo PK, Banks E. Epidemiology of breast cancer. Lancet Oncol. 2001;2: 133-140

[11] Forman D, Bray F, Brewster D.H. , et al. Cancer Incidence in Five Continents, Vol.X. In IARC Scientific Publications IARC: Lyon. 2014.

[12] Mogren I, Stenlund H, Högberg U. Long-term impact of reproductive factors on the risk of cervical, endometrial, ovarian and breast cancer. Acta Oncol. 2001;40: 849-854.

[13] Wernli KJ, Ray RM, Gao DL, De Roos AJ, Checkoway H, Thomas DB. Menstrual and reproductive factors in relation to risk of endometrial cancer in Chinese women. Cancer Causes Control. 2006;17: 949-955.

[14] Karageorgi S, Hankinson SE, Kraft P, De Vivo I. Reproductive factors and postmenopausal hormone use in relation to endometrial cancer risk in the Nurses' Health Study cohort 1976-2004. Int J Cancer. 2010;126: 208-216.
[15] Bevier M, Sundquist J, Hemminki K. Does the time interval between first and last birth influence the risk of endometrial and ovarian cancer? Eur J Cancer. 2011;47: 586-591.

[16] Albrektsen G, Heuch I, Tretli S, Kvåle G. Is the risk of cancer of the corpus uteri reduced by a recent pregnancy? A prospective study of 765,756 Norwegian women. Int J Cancer. 1995;61: 485-490.

[17] McPherson CP, Sellers TA, Potter JD, Bostick RM, Folsom AR. Reproductive factors and risk of endometrial cancer. The Iowa Women's Health Study. Am J Epidemiol. 1996;143: 1195-1202.

[18] Pfeiffer RM, Mitani A, Landgren O, et al. Timing of births and endometrial cancer risk in Swedish women. Cancer Causes Control. 2009;20: 1441-1449.

[19] Dossus L, Allen N, Kaaks R, et al. Reproductive risk factors and endometrial cancer: the European Prospective Investigation into Cancer and Nutrition. Int J Cancer 2010;127: 442-451.

[20] Fukao A, Tsubono Y, Komatsu S, et al. A Cohort Study on the Relation of Lifestyle, Personality and Biologic Markers to Cancer in Miyagi, Japan : Study Design, Response Rate and Profiles of the Cohort Subjects. J Epidemiol. 1995;5: 153-157.

[21] Tsuji I, Nishino Y, Ohkubo T, et al. A prospective cohort study on National Health Insurance beneficiaries in Ohsaki, Miyagi Prefecture, Japan: study design, profiles of the subjects and medical cost during the first year. J Epidemiol. 1998;8: 258-263.

[22] Sugiyama K, Sugawara Y, Tomata Y, Nishino Y, Fukao A, Tsuji I. The association between coffee consumption and bladder cancer incidence in a pooled analysis of the Miyagi Cohort Study and Ohsaki Cohort Study. Eur J Cancer Prev. 2017; 26: 125-130.

[23] Cramer DW. The epidemiology of endometrial and ovarian cancer. Hematol Oncol Clin North Am. 2012;26: 1-12.

[24] Xu WH, Xiang YB, Ruan ZX, et al. Menstrual and reproductive factors and endometrial cancer risk: Results from a population-based case-control study in urban Shanghai. Int J Cancer. 2004;108: 613-619.

[25] Setiawan VW, Pike MC, Karageorgi S, et al. Age at last birth in relation to risk of endometrial cancer: pooled analysis in the epidemiology of endometrial cancer consortium. Am J Epidemiol. 2012;176: 269-278. 